\title{
Numerical modeling of shape memory alloy problem in presence of perturbation: application to $\mathrm{Cu}-\mathrm{Al}-\mathrm{Zn}-\mathrm{Mn}$ specimen
}

\author{
Fatma Abid ${ }^{1,2,}{ }^{*}$, Abdelkhalak Elhami ${ }^{1}$, Tarek Merzouki ${ }^{3}$, Hassen Trabelsi ${ }^{2}$, Lassaad Walha ${ }^{2}$, and Mohamed Haddar $^{2}$ \\ ${ }^{1}$ Laboratory Of Mechanics Of Normandy (LMN), National Institute of Applied Sciences of Rouen, Rouen, France \\ ${ }^{2}$ Laboratory Of Mechanics, Modeling and Manufacturing (LA2MP), Mechanical Engineering Department, National School of \\ Engineers of Sfax, Sfax, Tunisia \\ ${ }^{3}$ Laboratory Of System Engineering Of Versailles, University Of Versailles Saint Quentin In Yvelines, Velizy, France
}

Received: 1 October 2018 / Accepted: 5 November 2018

\begin{abstract}
This paper proposes a methodology for taking into consideration uncertainties based on polynomial chaos (PC). The proposed approach is used in order to determine the response of $\mathrm{Cu}-\mathrm{Al}-\mathrm{Zn}-\mathrm{Mn}$ shape memory alloy specimen with uncertainties associated to material parameters. The simulation results are obtained by PC method. The proposed method seems to be an efficient probabilistic tool. It is worth mentioning that PC approach is an interesting alternative for the parametric studies. This technique is more efficient compared to MC approach.
\end{abstract}

Keywords: shape memory alloy specimen / material parameters / polynomial chaos method / uncertainty

\section{Introduction}

In recent decades, smart technologies become of increasing interest in different engineering fields [1]. The purpose is to develop new and intelligent systems that can be integrated with actuators, sensors and micro controllers. Shape memory alloy (SMA) is a smart material that is successfully used in the achievement of such technologies [2]. SMA material becomes more and more used due to its interesting physical and mechanical properties compared to other materials. Such material is characterized by the ability to remember its original shape after deformation. In fact, SMA can generate high values of thermal-mechanical driving forces and can undergo reversible moderate deformations up to $8 \%$ under loading/thermal cycles. Such a specific behavior of SMA is because of the native capability to undergo reversible changes of the crystallographic structure that depends on the temperature and on the state of the stress. These changes are due to the martensitic transformations between the crystallographic more ordered parent phase, the austenite, and the crystallographic less ordered parent phase, the martenite [3]. Generally speaking, shape memory alloy is a major challenge for the researchers due to its intelligent characteristics. In fact, the main attractive features of this class of materials are the capabilities of: (1) recovering

\footnotetext{
* e-mail: fatma.abid@insa-rouen.fr
}

the original shape after large deformations induced by mechanical load (pseudo-elasticity) and (2) maintaining a deformed shape up to heat induced recovery of the original shape (shape memory effect) [4]. Due to its special behavior, SMA is easily integrated in systems without causing a high increase in volume or weight. Besides, such material is directly activated by temperature cycles or stress [5]. These characteristics allow the SMA to be used in a wide range of engineering applications such as biomechanics such as surgical tool and prostheses, aeronautics and automotive. The study of shape memory alloy does not integrate dispersion in the shape memory alloy parameters. They are considered as constant. However, such parameters are uncertain due to their experiment measurement. Several methods are proposed in the literature for considering uncertainties. Monte Carlo (MC) method is well-known in this field but it is often costly because of the great numbers of samples required in the aim to have a reasonable accuracy [5-7]. Polynomial chaos method is also presented in the literature as an attractive probabilistic method for considering uncertainties.

The capabilities of such a method are demonstrated in biological and environmental problem [12], in solving partial and ordinary differential equations [13], in dynamic systems [5] and in parameter estimation [14]. The main contribution of this communication is the study of the uncertainty of $\mathrm{Cu}-\mathrm{Al}-\mathrm{Zn}-\mathrm{Mn}$ alloy. This communication is structured as follows: modeling of shape memory alloy specimen is presented in Section 2. One of the main 


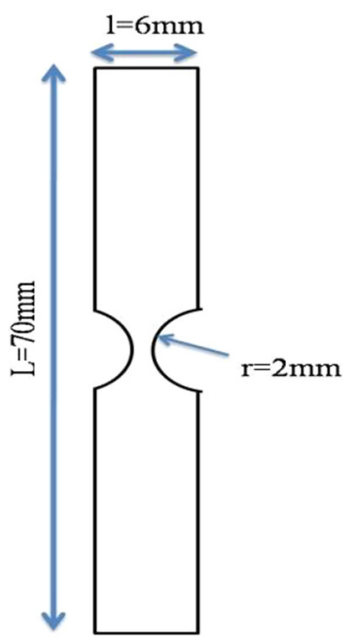

Fig. 1. 2D diagram of the specimen.

contributions of this work, the MC and PC methodology are fully in Sections 3 and 4. Finally, numerical results of shape memory alloy problem and comments are made based on the methodology carried out in this communication in Section 5.

\section{Modeling of $\mathrm{Cu}-\mathrm{Al}-\mathrm{Zn}-\mathrm{Mn}$ alloy specimen}

The considered model consists on a simple double notch specimen as shown in Figure 1. As a type of shape memory alloy, we choose the $\mathrm{Cu}-\mathrm{Al}-\mathrm{Zn}-\mathrm{Mn}$. The geometric characteristics of the studied specimen are the following: $l=6 \mathrm{~mm}, L=70 \mathrm{~mm}$ and $r=2 \mathrm{~mm}$. Numerical simulations of shape memory alloy response are performed using commercial software ANSYS.

Regarding the descritization of the specimen, 2D Plane 182 quadrilateral elements are used as shown in Figure 2. The $2 \mathrm{D}$ plane is formed by four node elements with four degrees of freedom at each node: 2 translations in the nodal $x, y$ directions and 2 rotations in the nodal $x, y$. The values of the materiel parameters used in this model are given in Table 1.

These parameters are taken from literature [15] and are respectively: the Young's modulus $E$, the Poisson's ratio $v$, the hardening parameter $h$, the reference temperature $T_{0}$, the elastic limit $R$, the temperature scaling parameter $\beta$ and the maximum transformation strain $\varepsilon_{l}$.

In this problem, the bottom of the specimen $(y=0)$ cannot be moved. The applied loading path of the $\mathrm{Cu}-\mathrm{Al}-$ $\mathrm{Mn}-\mathrm{Zn}$ specimen is shown in Figure 3. The first step $(A B)$ corresponds to the martensite variants orientation process. The second step $(B C)$ corresponds to the thermal loading above the austenite finish temperature Af. Steps 1-2 and 23 correspond to the heating and cooling of the specimen. In this study, the room temperature and the heating level are fixed to 225 and $500 \mathrm{~K}$.

\section{Monte Carlo (MC)}

In this part, the Monte Carlo method is described. This method refers to any calculation technique using successive

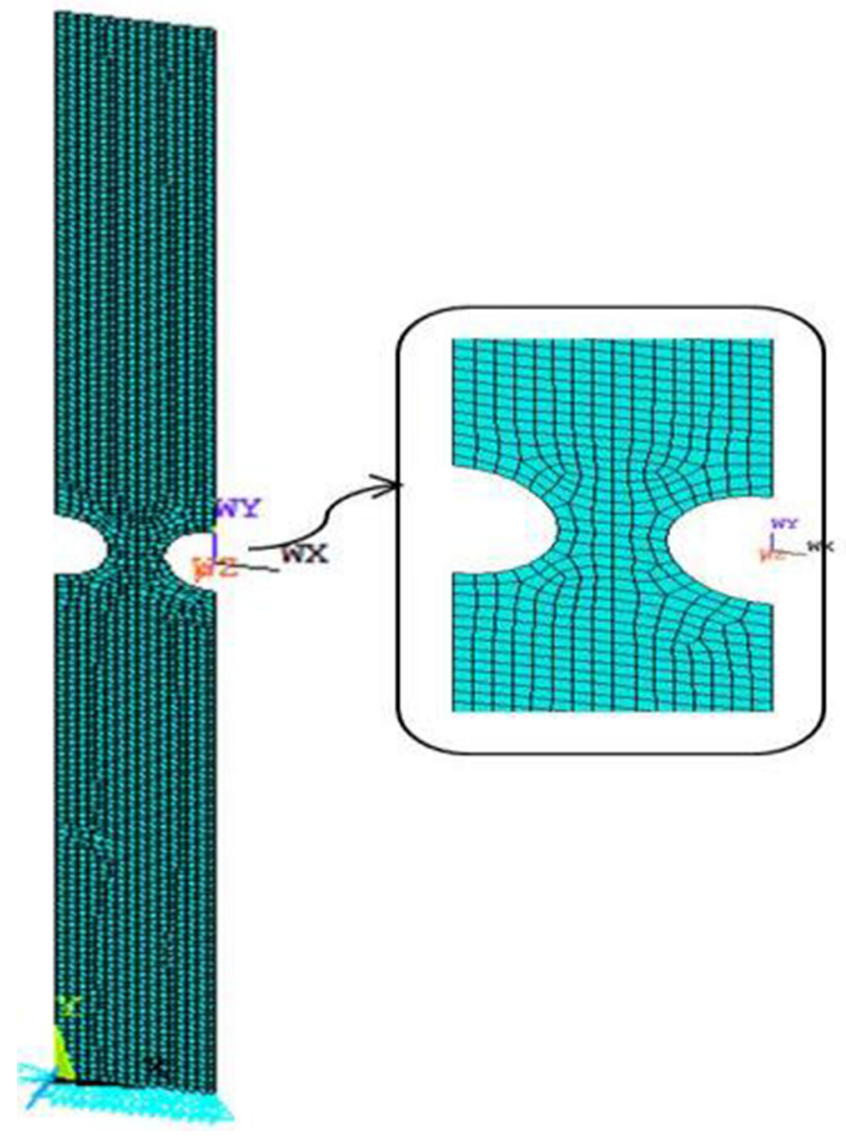

Fig. 2. ANSYS finite element model of the specimen.

Table 1. Material parameters.

\begin{tabular}{llllllll}
\hline Constant & $\begin{array}{l}E \\
(M P A)\end{array}$ & $v$ & $h$ & $\begin{array}{l}T_{0} \\
(M P a)\end{array}$ & $\begin{array}{l}R \\
(K)\end{array}$ & $\begin{array}{l}B \\
(M P a)\end{array}$ & $\mathcal{E}_{l}$ \\
\hline Value & 30700 & 0.36 & 9230 & 253.15 & 73.4 & 4.2 & 0.1 \\
\hline
\end{tabular}

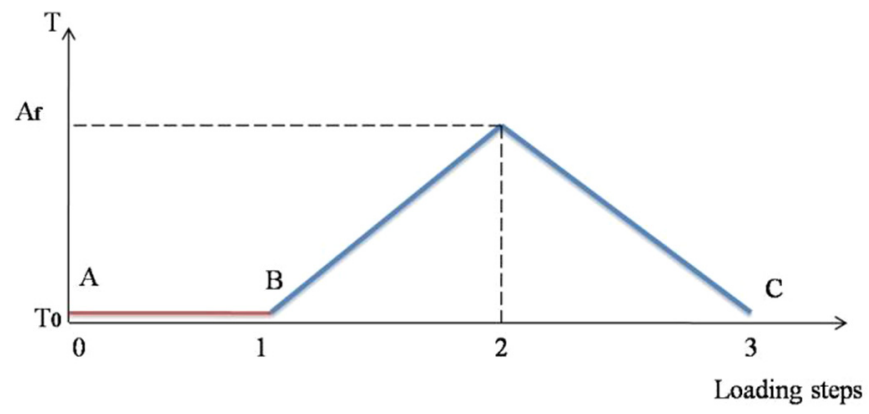

Fig. 3. Mechanical and thermal loading of the specimen.

resolutions of a deterministic system incorporating uncertain parameters, which are modeled by random variables. It is a powerful mathematical tool, which is used, in a wide range

Of applications. An $M C$ technique is used when the problem to be treated is complex for a resolution by analytic manner. It generates for all uncertain parameters 
and following their laws of probability and their correlations, random draws. For each draw, a set of parameters is obtained and a deterministic calculation, according to analytic or numerical models well defined, is operated [6]. This method can be applied to any system and the results are accurate. However, a reasonable accuracy needs a large number of draws. As a result, this method is expensive in term of computational time. Generally, the MC method is used as a reference method to validate the efficiency of others methods of uncertainty.

\subsection{Algorithm implementation}

The Monte Carlo method considers functions of the form:

$$
X=M(U),
$$

where $M$ represents the model under consideration, $U$ is the vector of uncertain input variables and $X$ is the vector of the estimated outputs. In fact, the $M C$ algorithm consists in five steps:

- the probabilistic identification of the uncertain parameters of the studied system;

- the sampling and the random generation of the achievements;

- spread of the uncertainty of the data set obtained by step 2 into the model and the determination of the corresponding output set;

- the estimation of the output distribution law;

- the convergence analysis of the distribution of the model output.

\section{Proposed method}

Different methods are used in order to model the propagation of uncertainty. These techniques are classified in three categories: simulation technique, perturbation technique and spectral technique. Monte Carlo $(M C)$ method is considered as a reference method in the calculation of system with uncertain parameters. The main problem of such a method comes from the high computational time which complicates the use of this technique. The perturbation technique considers the Taylor series development of the response around its mean. The main disadvantage of this method is the condition that ensures the convergence of these series. In fact, the variables must have low dispersion [8]. As a result, the method used in order to take into account uncertainty in this paper is the polynomial chaos (PC) method. The fundamental idea of PC, developed by Wiener [10] in 1938, is to separate the stochastic components of a random function and its deterministic components. In fact, the random process of interest is approximated by summing the orthogonal polynomial chaos of random independent variables [9]. The entire proposed methodology is described in the flowchart as shown in Figure 4. A brief mathematical review of this method is presented. For example, given any random variables $U_{i}$ such as the displacement in a shape

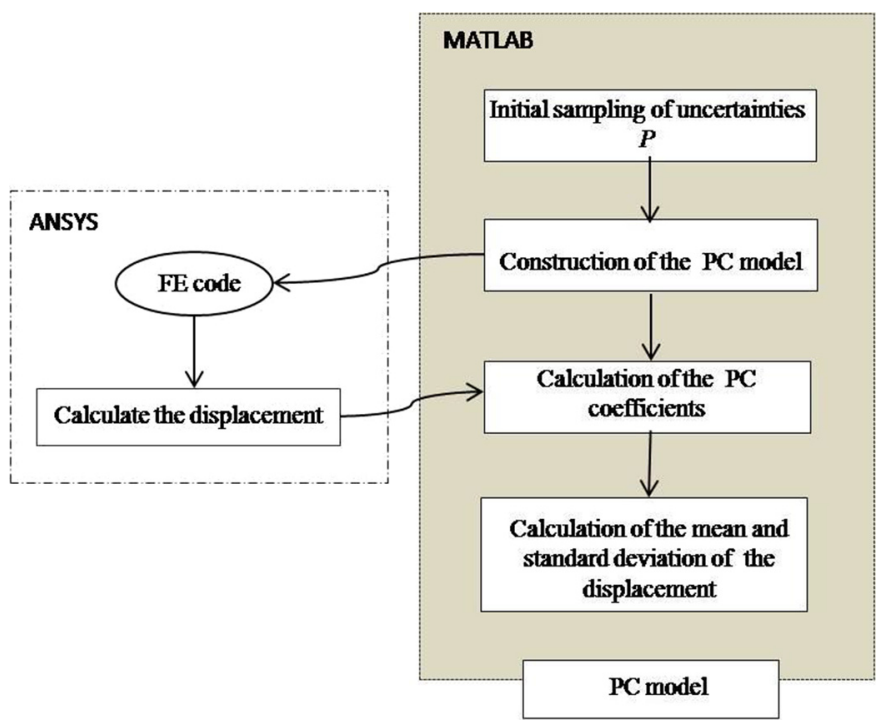

Fig. 4. Flowchart of the PC methodology.

memory alloy system, we can write as follows [11]:

$$
U_{i, j}(u, \xi)=\sum_{j=0}^{\infty} \bar{U}_{i, j}(x) \psi_{j}(\xi),
$$

where $\xi$ is a vector of standard normal random variables, $U_{i, j}$ is the deterministic component and $\psi_{j}(\xi)$ is the orthogonal polynomials such as Legendre, hermite, etc. The choice of the polynomial family is determined by the density distribution of the uncertain input parameter. As a result, a correspondence of the families of orthogonal polynomials and the families of probability laws is established. Because that a series expansion to infinity cannot be used in practice, the sum is truncated to an order $N_{p}$ in order to limit the number of terms to finite ones. The order $N_{p}$ depends on the dimension $r$ of the polynomial chaos and its order $p$. We can write then:

$$
U_{i}(x, \xi)=\sum_{j=0}^{N_{p}} U i, j(y) \psi_{j}(\xi)
$$

with:

$$
N_{p}=\frac{(p+r) !}{p ! r !}-1 .
$$

The calculation of the representation by the $P C$ method requires the determination of $N_{p}+1$ stochastic components. The following step is to determine the PC coefficients by regression approach or spectral projection technique (NISP).

\section{Numerical results and discussions}

In this section, the analysis of the shape memory alloy specimen is performed with and without uncertainties. 


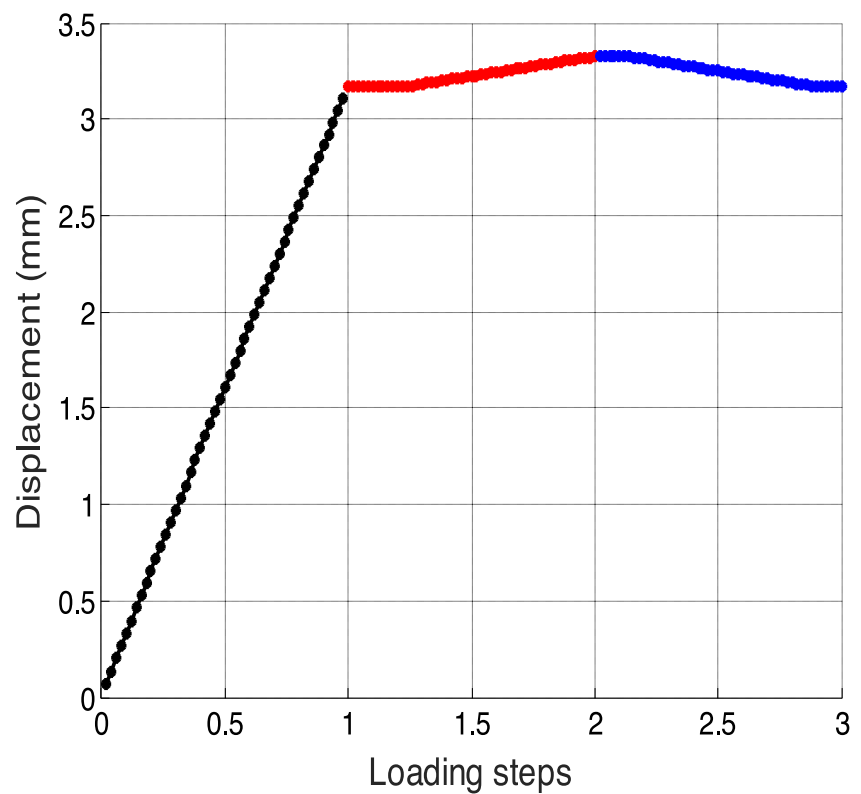

Fig. 5. Displacement during the steps 0-3. a: black line corresponding to mechanical load; b: red line corresponding to heating load; c: blue line corresponding to cooling load.

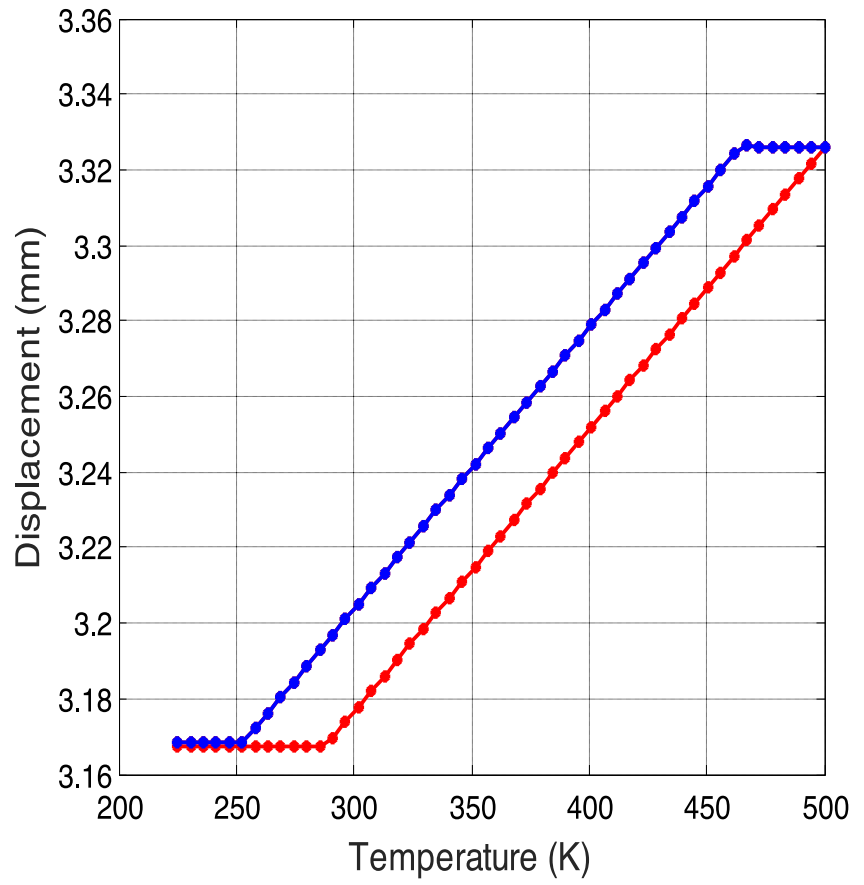

Fig. 6. Displacement as a function of heating. a: red line corresponding to heating load; b: blue line corresponding to cooling load.

\subsection{Deterministic analysis}

Figure 5 shows the shape memory alloy specimen during the steps (A-B) and (B-C) which are presented in Figure 3. Figure 6 gives the displacement as a function of applied temperature.

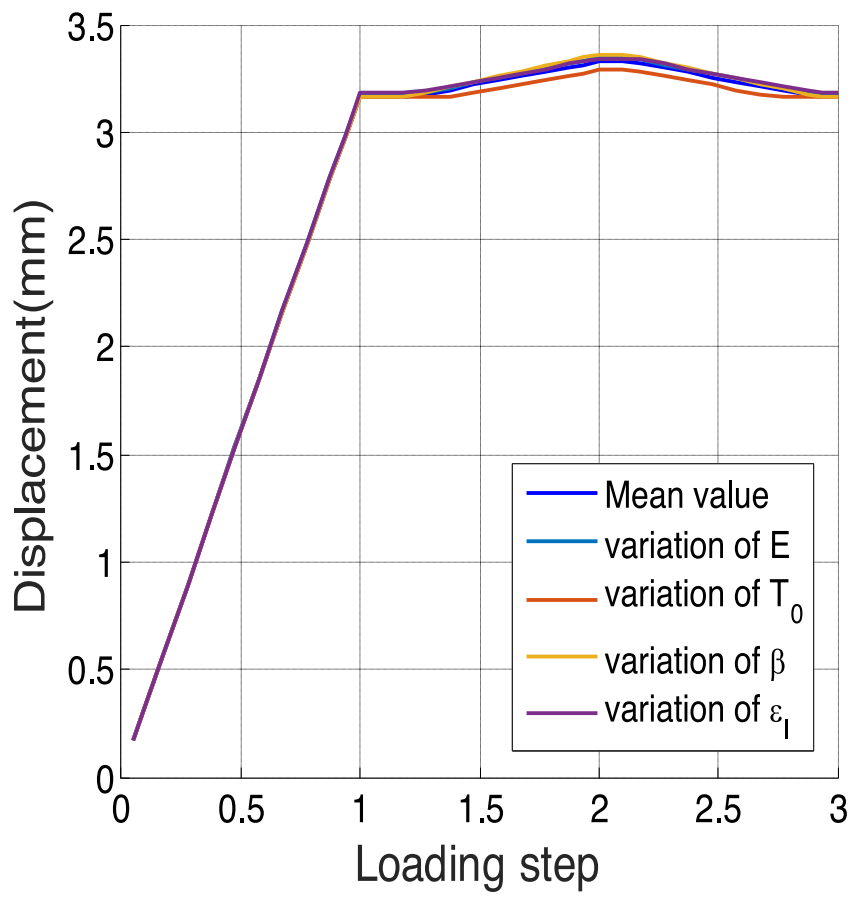

Fig. 7. Variables with influence in the displacement.

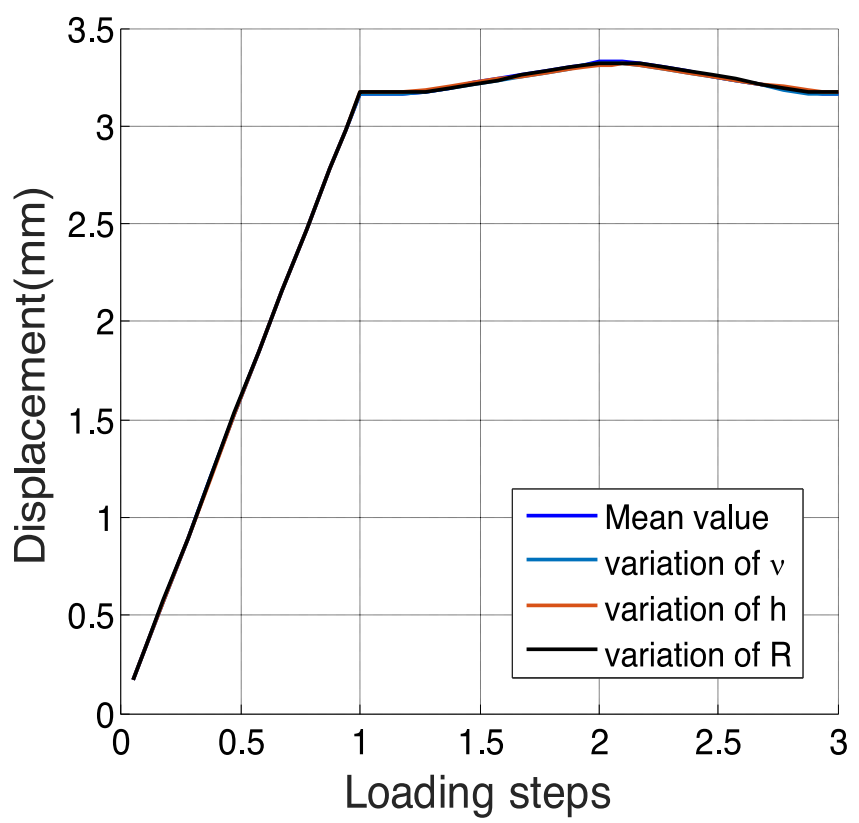

Fig. 8. Variables with non influence in the displacement.

During the loading step, the temperature is kept constant at $T=225 \mathrm{~K}$. For the step 2, the specimen is gradually heated to $500 \mathrm{~K}$. After that, the temperature has returned to the room temperature. It seems that the specimen starts to move at a temperature equal to $285.5 \mathrm{~K}$. This corresponds to the reverse transformation from martensite to austenite. The cooling allows it to return to a martensitic state. 


\subsection{Sensitivity analysis}

The purpose of this part is to study the sensitivity of the $\mathrm{Cu}-\mathrm{Al}-\mathrm{Zn}-\mathrm{Mn}$ specimen response to input variables. Figure 7 represents the influence of the first set of physical parameters on the specimen considering a standard

Table 2. Characteristic of uncertain parameters.

\begin{tabular}{llllc}
\hline Constant & $E(M P A)$ & $T_{0}(K)$ & $B\left(M P a K^{-1}\right)$ & $\mathcal{E}_{l}$ \\
\hline Value & {$[24560 ;$} & {$[202.52 ;$} & {$[3.36 ;$} & {$[0.08 ;$} \\
& $36840]$ & $303.78]$ & $5.04]$ & $0.12]$ \\
\hline
\end{tabular}
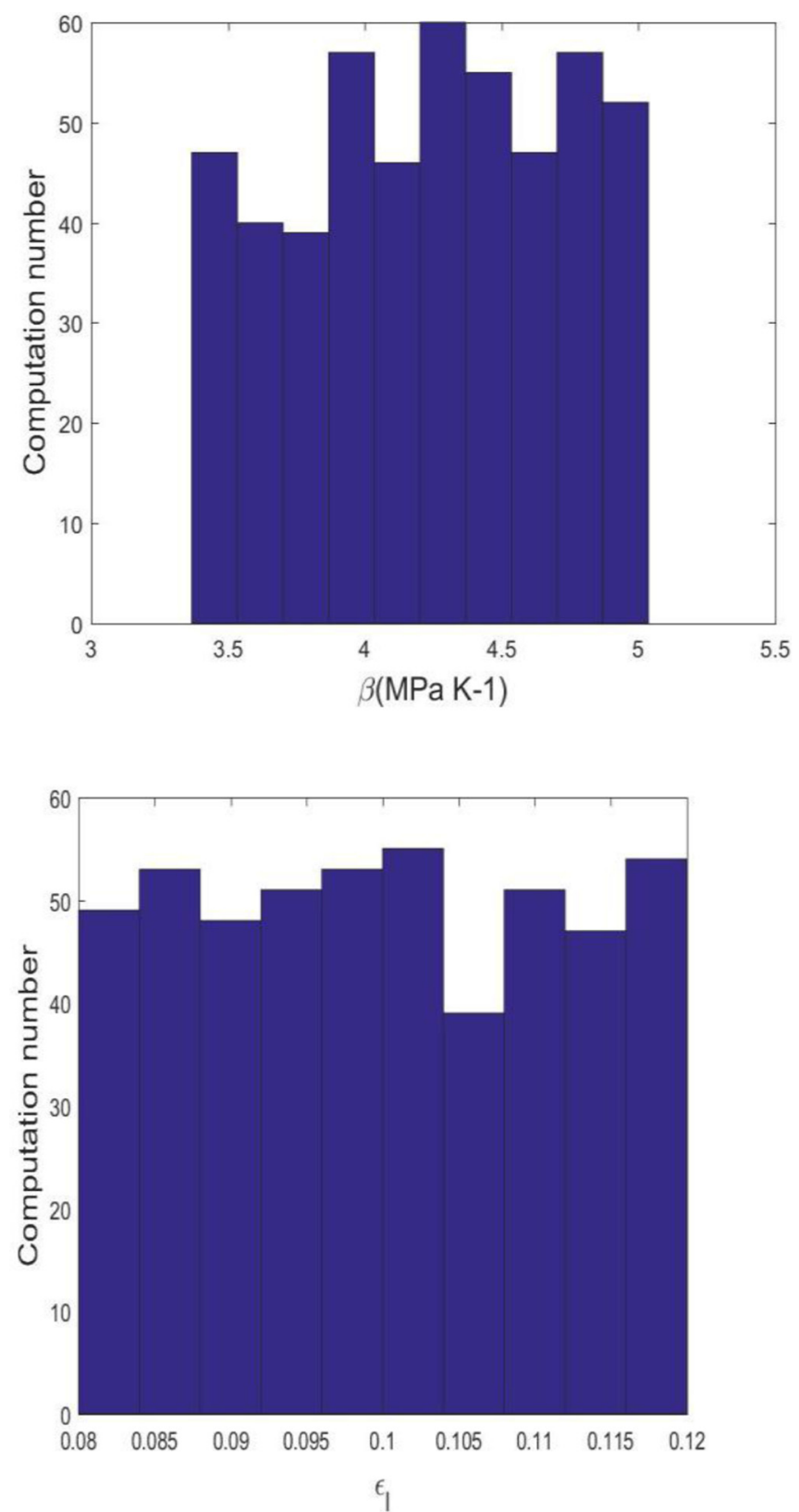

deviation of $20 \%$. The set is formed by the Young's modulus $E$, the reference temperature $T_{0}$, the temperature scaling parameter $\beta$ and the maximum transformation strain $\mathcal{E}_{l}$. By comparing these curves with the mean value curve, it can be clearly seen that these parameters have an influence on the behavior of the studied example. Figure 8 shows the displacement of the specimen considering the other set of the physical parameters: Poisson's ratio $v$, the hardening parameter $h$ and the elastic limit $R$. From Figure 8, we can conclude that these variables have a weak influence on the behavior of the specimen. In the next subsection, we will take into account the parameters that have an influence on the behavior of the studied specimen.
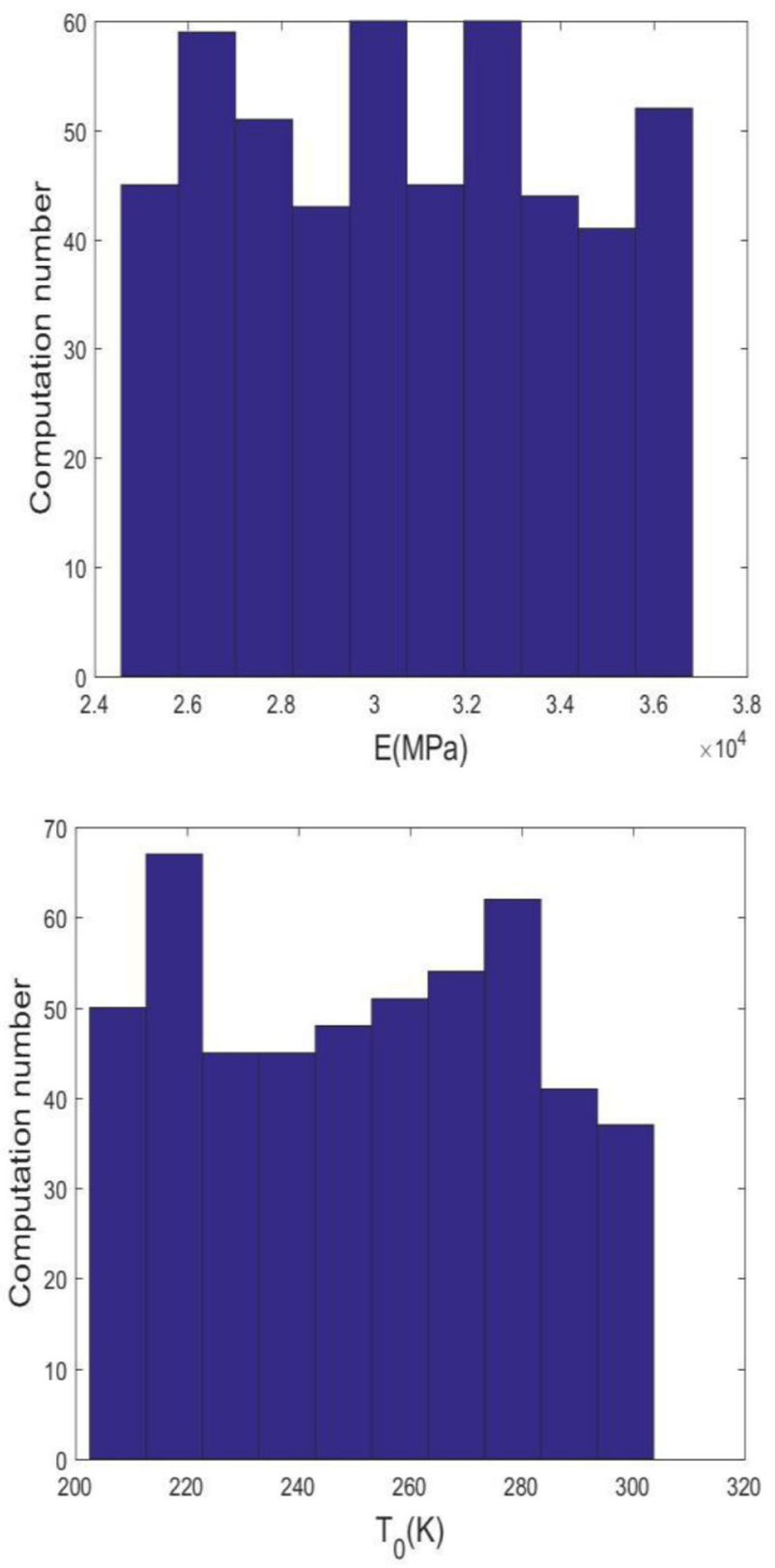

Fig. 9. Probability distribution of the inputs. a: the Young's modulus $E$; b: the temperature scaling parameter $\beta$; c: the reference temperature $T_{0}$; d: the maximum transformation strain $\varepsilon_{l}$. 


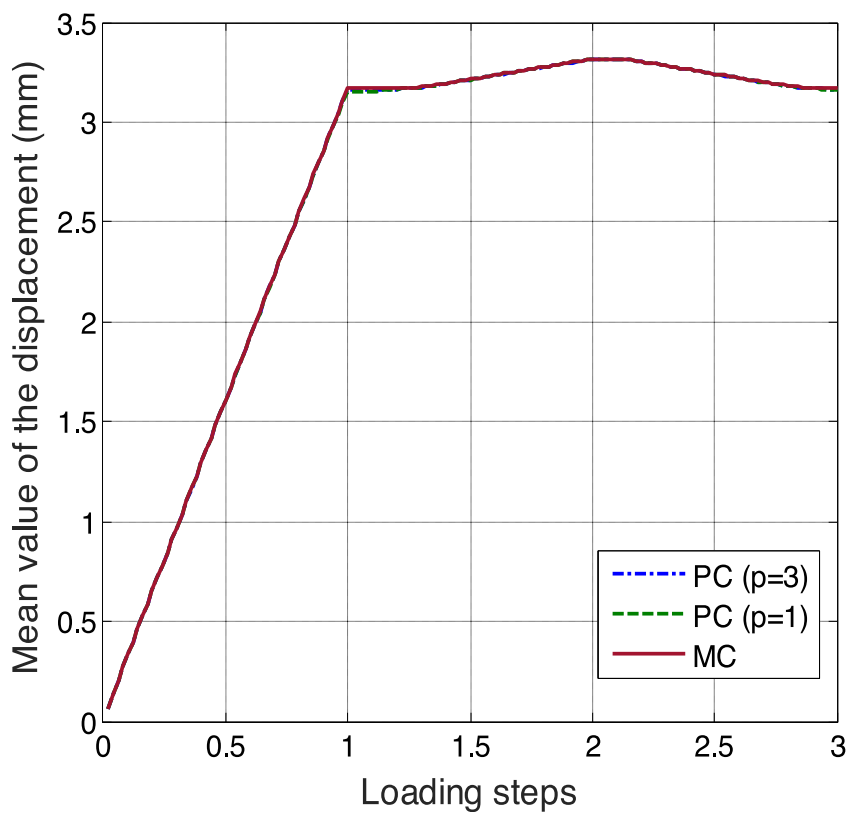

Fig. 10. Mean value of the displacement.

\subsection{Probabilistic analysis}

In this part, the static behavior of $\mathrm{Cu}-\mathrm{Al}-\mathrm{Zn}-\mathrm{Mn}$ specimen is investigated using polynomial chaos $(P C)$ approach. The $P C$ results are compared with the Monte Carlo $(M C)$ method. The material parameters of such a studied system are summarized in Table 1. Uniform probability distribution is treated in order to describe the random parameters. In this case, the Legendre polynomials are the best used to deal with uniform uncertainties. They are calculated using the recurrence relation as mentioned in the equation:

$$
\left\{\begin{array}{l}
P_{L_{0}}(u)=1 \\
P_{L_{1}}(u)=u \\
(n+1) P_{L_{n+1}}(u)=(2 n+1) u P_{L_{n}}(u)-n P_{L_{n-1}}(u)
\end{array} .\right.
$$

Numerical results are presented for the formulation derived in Section 3. The material variables of the $\mathrm{Cu}-\mathrm{Al}-$ Zn-Mn specimen that influence in the displacement are respectively: the Young's modulus $E$, the temperature scaling parameter $\beta$, the reference temperature $T_{0}$ and the maximum transformation strain $\varepsilon_{l}$. These variables are supposed to be random and they are defined as shown in Table 2. The parameters are chosen to be random following a uniform distribution around their normal values $\pm 20 \%$.

Using the Monte Carlo approach consists on creating a grid of numerical values from the certain parameters and calculating the quantity of interest. The quantity of interest is analyzed for 500 simulations. Figure 9 represents the distribution of the input variables $\left(E, \beta, T_{0}, \varepsilon_{l}\right)$ in the case of uniform distribution. Figures 10 and 11 present the evolution of the displacement as a function of loading steps by two stochastic approaches $M C$ and $P C$ in the case of 4 uncertain parameters and for two values of $P C$ orders.

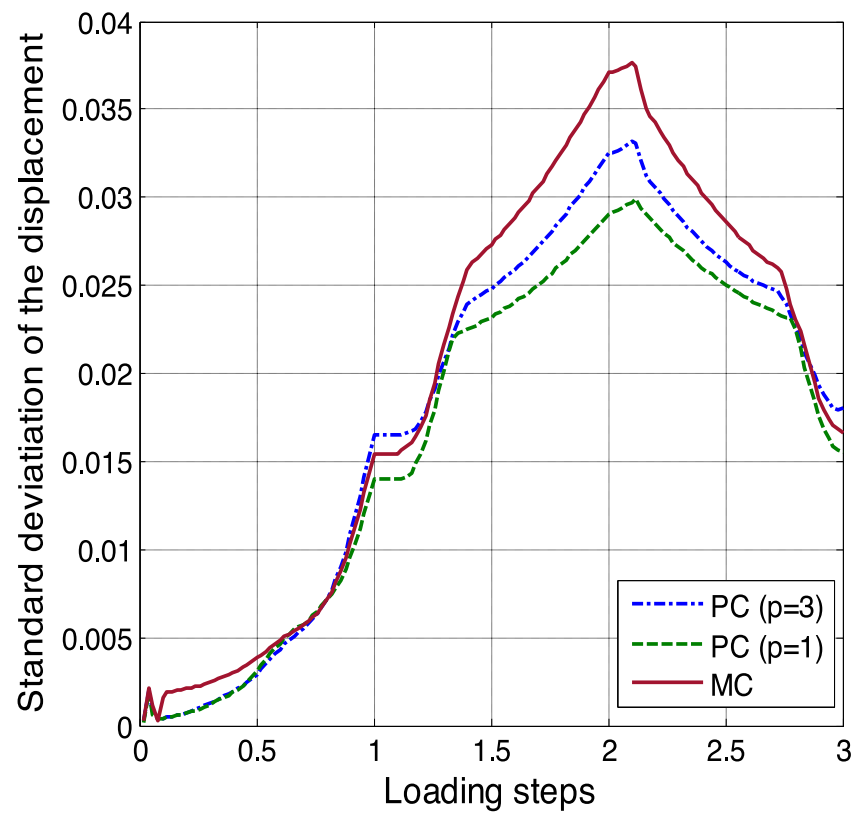

Fig. 11. Standard deviation of the displacement.

Table 3. Summary results of the displacement.

\begin{tabular}{ll}
\hline Number of uncertain parameters & $r=4\left(E, \beta, T 0, \varepsilon_{l}\right)$ \\
\hline$N_{M C}$ & 500 \\
Order of chaos & 3 \\
Mean maxi of the displacement & $3.317 / 3.316$ \\
(MC, PC) & \\
Std maxi of the displacement & $0.037 / 0.033$ \\
(MC, PC) & \\
Time MC (h) & 85.208 \\
Time PC (h) & 33.39 \\
\hline
\end{tabular}

The mean value and the standard deviation of the displacement of the specimen are calculated by polynomial chaos approach. The obtained results are compared with those given from $M C$ simulations for 500 simulations. Figures 10 and 11 show the mean value and the standard deviation of the displacement of the specimen in the case of $p=1$ and $p=3$. It can be seen from these figures that as $p$ increases, the result seems to become better. For $p=3$, the displacement of the specimen matches with the $\mathrm{MC}$ simulations results. These figures show that the obtained solutions are around the Monte Carlo simulation which is the reference solution. Besides, one can notice that the computational time is considerably reduced. Table 3 presents a summary of the two stochastic methods used in this example.

It is worth mentioning that the Monte Carlo approach is a well-known technique used in order to solve complex system with uncertainties. To have a reasonable accuracy, this method requires a great number of samples. In this 
paper, 500 of sampling of 4 input variables are calculated and then the problem is solved for each sample of input variables.

However, this approach has a poor convergence for the mean and the standard deviation of the solution. Thus, it requires a large number of samples to have a good precision. The $P C$ method is used as an alternative to deal with the uncertainty, quantification. Such a method is more efficient compared to $M C$ method.

\section{Conclusion}

In this work, the Monte Carlo method and the $P C$ approach were coupled to finite element solutions discussed above in order to calculate the displacement of the $\mathrm{Cu}-\mathrm{Al}-\mathrm{Zn}-\mathrm{Mn}$ specimen. The response of such a material is coupled to probabilistic approaches when material parameters present uncertainties. Results using $P C$ are compared with $M C$ method. Convergence was verified with comparisons against solution from $M C$ simulations. The main results of the present study demonstrate that the PC method may be an effective alternative of $M C$ simulations. As regard efficiency, the $P C$ based simulation is computationally less expensive compared to $M C$ in order to generate solutions. A future track of this work is to apply optimization under uncertainty in complex system formed by shape memory alloy. Further work in this context is in progress.

The present research work has been supported by the laboratory of mechanics of normandy (LMN), INSA Rouen and the laboratory of mechanics, modeling and manufacturing (LA2MP), ENI Sfax. The authors gratefully acknowledge then support of these institutions

\section{References}

1. D.C. Lagoudas (Ed.), Shape memory alloys: Modeling and engineering applications (Springer-Verlag, 2008)

2. T. Merzouki, A. Duval, T.B. Zineb, Finite element analysis of a shape memory alloy actuator for a micropump, Simul. Model. Pract. Theory 27, 112 (2012)
3. P. Bisegna, F. Caselli, S. Marfia, E. Sacco, A new SMA shell element based on the corotational formulation, Comput. Mech. 54(5), 1315 (2014)

4. A. Alipour, M. Kadkhodaei, A. Ghaei, Finite element simulation of shape memory alloy wires using a user material subroutine: Parametric study on heating rate, conductivity, and heat convection, J. Intell. Mater. Syst. Struct. 26(5), 554 (2015)

5. A. Guerine, A. El Hami, L. Walha, T. Fakhfakh, M. Haddar, Dynamic response of a spur gear system with uncertain parameters, J. Theor. Appl. Mech. 54(3), 1039 (2016)

6. K. Dammak, A. El Hami, S. Koubaa, L. Walha, M. Haddar, Reliability based design optimization of coupled acousticstructure system using generalized polynomial chaos, Int. J. Mech. Sci. 134, 75 (2017)

7. K. Dammak, S. Koubaa, A. El Hami, L. Walha, M. Haddar, Numerical modelling of vibro-acoustic problem in presence of uncertainty: Application to a vehicle cabin, Appl. Acoust. 144, 113 (2019)

8. M. Beyaoui, M. Tounsi, K. Abboudi, N. Feki, L. Walha, M. Haddar, Dynamic behaviour of a wind turbine gear system with uncertainties, Comptes Rendus Méc. 344(6), 375 (2016)

9. K. Sepahvand, M. Scheffler, S. Marburg, Uncertainty quantification in natural frequencies and radiated acoustic power of composite plates: Analytical and experimental investigation, Appl. Acoust. 87, 23 (2015)

10. N. Wiener, The homogeneous chaos, Am. J. Math. 60(4), 897 (1938)

11. L. Nechak, S. Berger, E. Aubry, Robust analysis of uncertain dynamic systems: Combination of the centre manifold and polynomial chaos theories, WSEAS Trans. Syst. 9(4), 386 (2010)

12. S.S. Isukapalli, A. Roy, P.G. Georgopoulos, Stochastic response surface methods (SRSMs) for uncertainty propagation: Application to environmental and biological systems, Risk Anal. 18(3), 351 (1998)

13. M.M.R. Williams, Polynomial chaos functions and stochastic differential equations, Ann. Nucl. Energy 33(9), 774 (2006)

14. G. Saad, R. Ghanem, S. Masri, Robust system identification of strongly non-linear dynamics using a polynomial chaosbased sequential data assimilation technique, in: 48th AIAA/ ASME/ASCE/AHS/ASC Structures, Structural Dynamics, and Materials Conference, 2007, p. 2211

15. N. Motte, A study to evaluate non-uniform phase maps in shape memory alloys using finite element method, Doctoral dissertation, Virginia Commonwealth University, 2015

Cite this article as: Fatma Abid, Abdelkhalak Elhami, Tarek Merzouki, Hassen Trabelsi, Lassaad Walha, Mohamed Haddar, Numerical modeling of shape memory alloy problem in presence of perturbation: application to Cu-Al-Zn-Mn specimen, Int. J. Simul. Multidisci. Des. Optim. 10, A7 (2019) 